\title{
Síndrome de Fisura Media del rostro
}

\author{
Dra. Silvia Castillo T. 1 . Dr, Ronald Youlton R.2, \\ Dr. lorge Rojas Z. 3 y Sra. Lucía Rivera A. ${ }^{4}$
}

\section{Median Cleft Face Syndrome}

\begin{abstract}
Minumal diagnostic criteria for the diagnosis of the unedian ofeft face syndrome are: true ocular lypertelorism, broad nisat root, median filciat groove or true cleftung. Involving the nose, the upper lip and palate and anterior cranium bifidum ocultum. We report the clinical findings in one fetmale and four male patients, aged 9 months to 16 years, in whom this diagnosis was establisbed. Chromosome studies were normal. 'l'heir family histories do not suggest an autosomic dominant or autosomic recesive mode of inheritance. It is possible that the genesis of the syndrome is multifactorial.
\end{abstract}

En nuestro medio, la incidencia de labio leporino con o sin fisura palatina es I por cada 642 nacidos vivos ${ }^{1}$. Con cierta frecuencia se observan otras anomalias asociadas, configurándose en algunos casos sindromes bien caracterizados ${ }^{2}$. Uno de ellos es el síndrome de fisura media facial o displasia frontonasal, del que hemos tenído la oportunidad de ver 5 casos que son el motivo de esta publicación.

\section{Casus Clínicos}

Cinco pacientes de 9 meses a 16 años de edad, cuatro de ellos varones, consultaron en el Policlinico de Cirugia del Hospital $R$. del Ríc y en el Servicio de Genética del Hospital J.J. Aguirre. Las genealogías fueron confeccionadas en base a información proporcionada por ios padres. Los niños, sus padres $y$ hermanos, literon examinados por uno de los autores. En todos se midieron las distancias interpupilar e intercantales, para calcular el índice cantal ${ }^{11-13}$

En cuatro de ellos se hizo estudio cromosómjco de linfocitos circulantes; el análisis se realizo con tinción para bandas $G{ }^{33}$

\section{Caso 1.}

C.A.L.L. Paciente varón, de 9 meses, nacido de embarazo normal y de término. Peso de nacimiento: 3850 grs. Desarrollo psicomotor y creci-

Trabajo de ingreso a la Sociedad de Genética de Chile, presentado en la XV Riunión Anual de la Sociedad de Genética de Chile, Punta de Tralca, Noviembre 1982.

${ }^{1}$ Becada, Servicio de Genćrica, Hospital \$.J. Aguirre, U. de Chile.

2 Pediatra, Servicio de Genétiea, Hospital J.J. Aguirre.

${ }^{3}$ Cirujano Infantil, Depto. de Cirugía, Hospital R. del Rio.

4 Asistente Social, Servicio de Genética, Hospital J.J. Aguirre. miento normales. La madre trabaja y lo hizo durante el embarazo en un laboratorio farmacéutico. en contacto con extracción de drogas. Los padres $y$ un hermano mayor son normales. Examen físico: Fontanela anterior continúa a frontal, se palpa un hueso wormiano central. Agenesia de piel en zona media. Alteración en implan. tación del pelo, en $V$ esbozado. Prominencia bifrontal. Telecanto primario. Hipertelorismo. Microftalmia derecha. Cejas con distribución en abanico. Fisura de ala nasal a derecha. Labio leporino bilateral, fisura velopalatina. Resto del examen: pliegues palmares anómalos. $P_{i e}$ bot bilateral. Cariotipo: $46, \mathrm{XY}$.

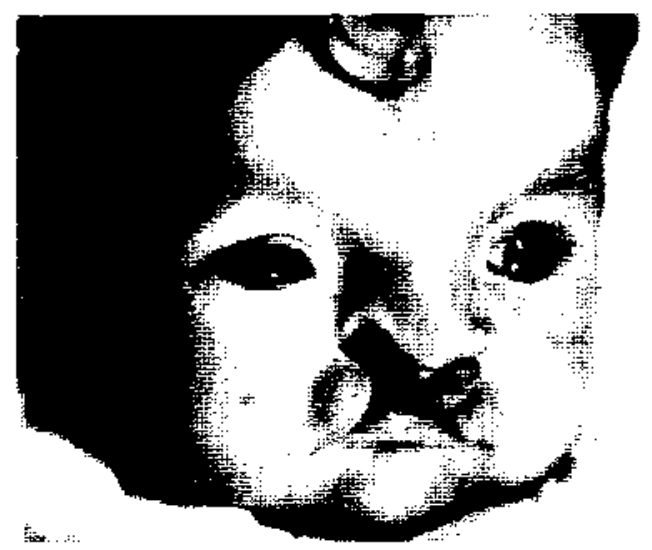

Figura I

\section{Caso 2.}

J.T.A. Paciente de sexo femenino, 8 afios, nacida de embarazo normal y parto a los 9 meses. Peso de nacimiento: 2600 grs. Desarrollo psicomator y crecimiento normales. Cursa $2^{\circ}$ año básico con regular rendimiento. Padres sanos. Hija única después de dos abortos espontáneos. Proce- 
de de Ancud. Una tía bisabuela fallecida habria sido portadora de fisura palatina. Examen físico: Implantación irregular del pelo en la frente con descenso en zona media derecha. Ceja derecha con remolino en extremo interno. Hipertelorismo. Dermoides epibulbares bilaterales con adherencias hacia párpado. Nariz ancha, sin punta. Narinas antevertidas. Labio leporino bilateral operado con fisura palatina central y compromiso de la premaxila. Micrognatia. Papilomas preauriculares bilaterales. Resto del exanen normal. Cariotipo: no se ha estudiado.

\section{Caso 3. -}

J.S.S. Paciente de sexo masculino, 16 años, nacido de embarazo y parto sin incidentes. Desarrollo psicomotor y crecimiento normales. Cursi $2^{\circ}$ año medio con buer: rendimiento. Padres $y$ hermana mayor normales. ]a madre tuvo dos abortos espontáneos, uno antes y otro después de él. Una prima de quinto grado de parentesco ten. dria un labio y paladar fisurados. Examen físico: Implantación en $V$ del pelo en la línca media con dos descensos menores bilaterales. Hipertelorismo. Nariz de base ancha, al nacer plana: actual. mente levantada con cícatriz quirúrgica en todo el dorso y columela. En el acto operntorio se observó una fisura media del tabique nasal hipoplásico. Boca con arco de cupido pronunciado. Paladar ojival. Restu del examen normal. Carioti. po: $46 . X Y$.

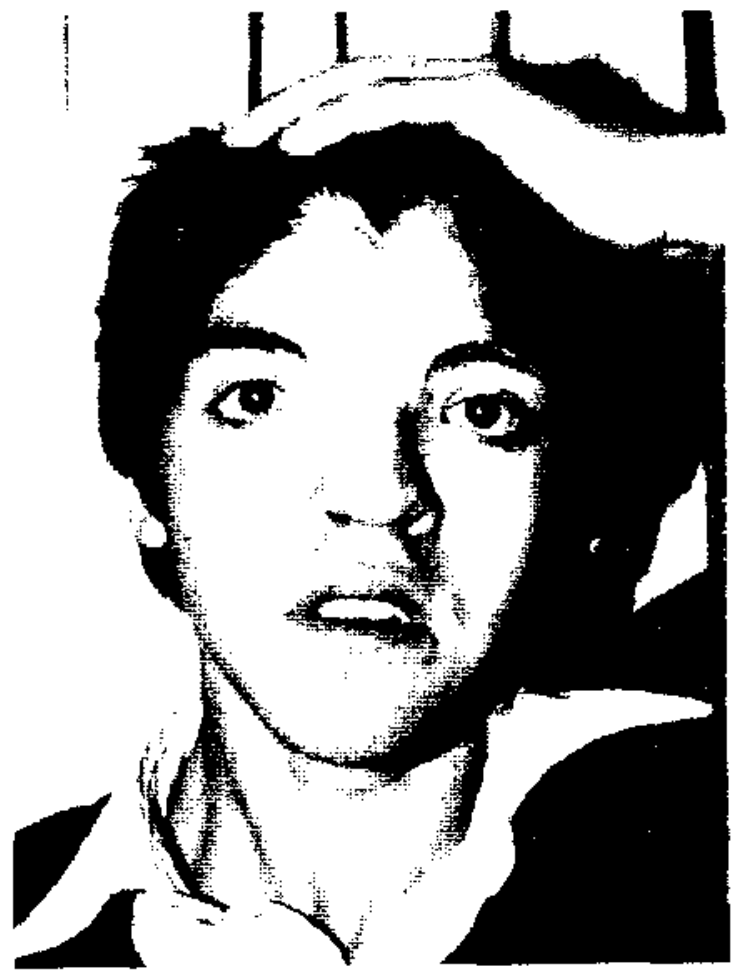

Figura 2

\section{Caso 4.}

R.B.T. Paciente de sexo masculino, 16 años, que nace de parto normal. Crecimiento adecuádo. Padres y tres hermanos sanos y no hay ante. cedentes familiares de anomalia sintilar. Examen fisico: Frente plana. Hipertelorismo. Puente nasal ancho y deprimido, fistula del dorso sin punta nasal. Paladar ojival. Sin otras alteracioncs. Retardo mental definido: $\mathrm{CI}=62$. Cariotipo: $46 . \mathrm{XY}$.

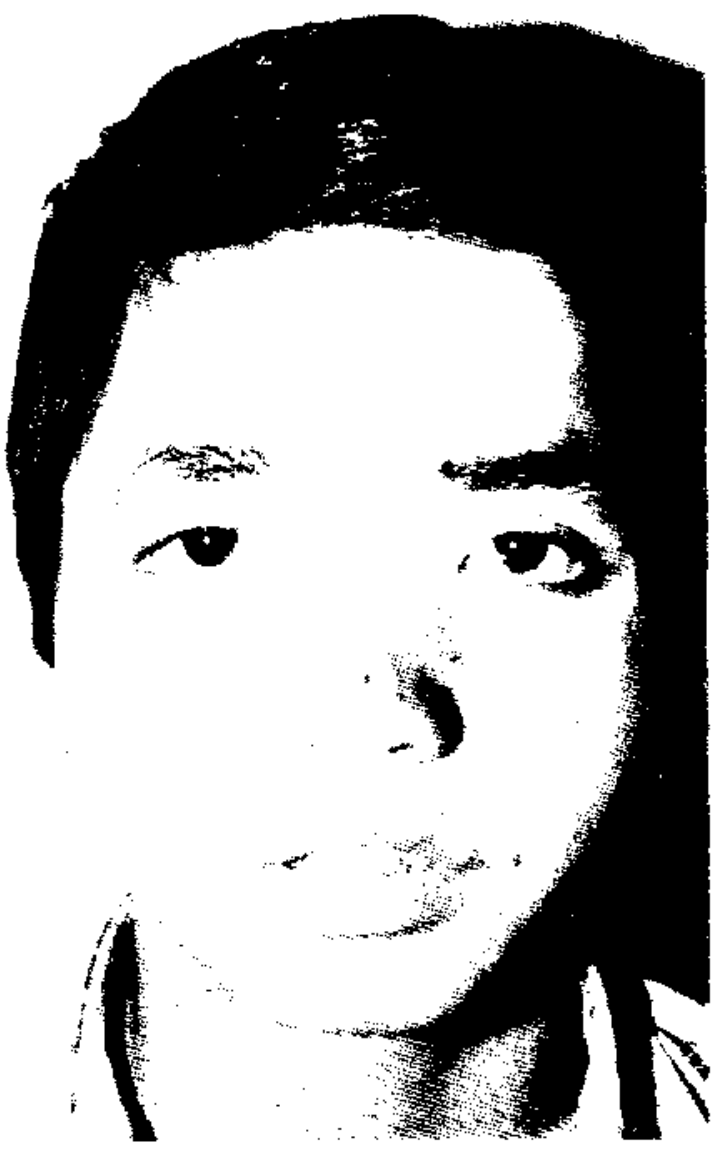

Figura 3

Caso 5 .

J.M.S. Paciente de sexo masculino, 3 afios, nacido de parto prematuro a los 6 meses con asfixia neonatal grave y problemas respiratorios que lo hacen estar hospitalizado durante 3 meses. Peso de nacimiento: 1250 grs. Desarrollo psicomotor Lento. Crecimiento normal. Padres y hermanos snos, antecedente de un aborto espontáneo o previo a su nacimiento. Examen físico: Frente prominente. Hipertelorismo. Puente nasal bajo, surco vertical en cuarto distal de la nariz. Hipoplasia del esmalte. Resto del examen: surco simiano en mano derecha. Retardo psicomotor moderado. Cariotipo: 46, XY. 


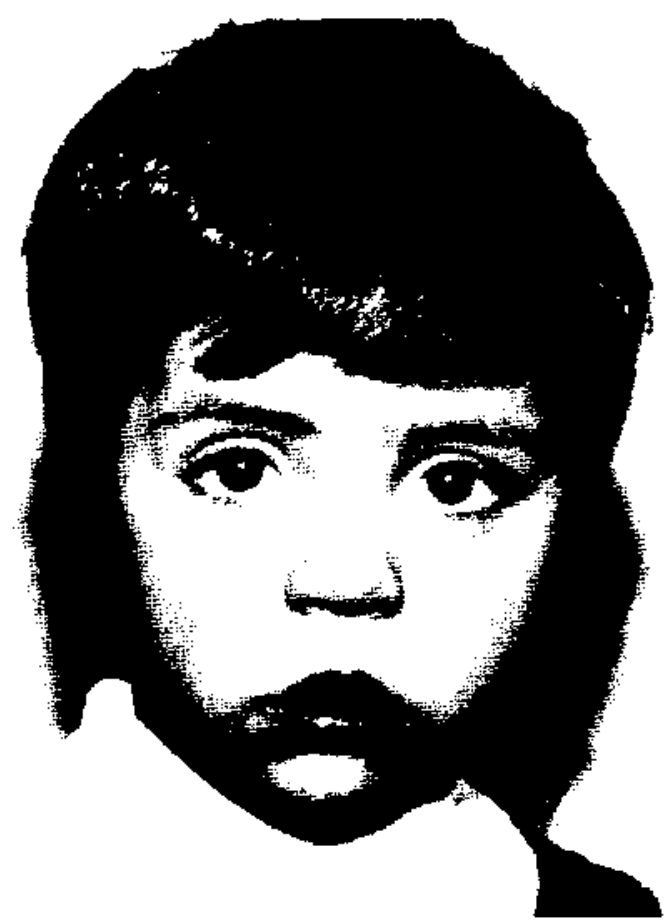

Figura 4

\section{DISCUSION}

Para una mejor comprensión de la variabilidad de este sindrome, es indispensable recordar la embriología de la cara ${ }^{3 \cdot 4-5}$

El cierre del tubo neural en el primer mes de vida es crítico para el desarrollo craneofacial, el que se inicia por migración de las células de la cresta neural. A diferencia del resto del cuerpo, cl mesénquima craneofacial deriva del ectodermo.

En el embrión de $6 \mathrm{~mm}$. de longitud ápico caudal, al $33^{\circ}$ día de vida, se encuentra uл engrosamiento epitelial, la placoda nasal u olfatoria, a ambos lados de la región frontal, por encima del estomodeo. Inicialmente son convexas, luego comienzan a hundirse pata formar el surco olfa. torio. Este hundimientu se debe a la elevación del ectodermo que lo rodea, producido por una proliferación del mesénquima adyacentc. Estas elevaciones son más acentuadas a ambos lados de la fosita y se denominan pliegue nasal interno y lateral. El pliegue nasal interno, junto con la región intermedia situada por encima del estomodeo, forma el proceso frontonasal. El proceso nasal externo separa al ojo. Por debajo, están los procesos maxilares y mandibular, derivados del primer arco faringeo y primer arco branguial respectivamente, separados por el estomodeo. El proceso frontonasal y los procesos maxilar y mandibular de cada lado son los elementos a partir de los cuales se desarrolla la cara.
En este período, ambos procesos nandibula. res se fusionan en la linea media. Cada proceso maxilar crece hacia adelante, se une al borde inferior lateral del pliegue nasal lateral $y$ se extiende por debajo del orificio anterioz, hasta alcanzar ei proceso nasal medio, con cl que se fusiona. ET orificio del estomodeo disminuvo en sentido transversal a causa de la fusion de las porciones de los procesos que van a formar las mejillas. Todos los surcos situados entre los distintos procesos faciales desaparecen normaimente en jos embriones de 45 dias. $30 \mathrm{~mm}$ de longitud $\mathrm{AC}$.

El ejc orbital se mueve hacid adelante de $180^{\circ}$ en el $2^{\circ}$ mes fetal a $71^{\circ}$ al nacimients. El epitelo olfatorio induce la formación de la cápsula nasa! cartilaginosa que es fundemental para completa: la migración hacia central. A los 60 dias, $30 \mathrm{~mm}$. de longitud $\mathrm{AC}$, la nariz esta representada por una prominencia bien definida, los labios supefior e inferíor estan completos; mejillas, párpados y el oído externo reconocibles. La cara ha alcanzado las caracteristicas de un rostro humanc.

Los factores que controlan la diferenciación del condrocráneo durante el periodo embriónico parccen ser determinados genéticamente y esta* rían sujetos a una influencia ambiental minima. Después de la diferenciación inicial de los tipos celulares específicos, el crecimiento depende más de factores epigenćticos y locales. Según sea el momento en que actúe la noxa serả el grado y tipo del defecto. Ilabía un defecto en la extensión anterior normal del ectornesénquima de la crcsta neural sobre los procesos frontonasales. Algún obstáculo impediría la migración de las cêlulas crestales.

Se discute si habría pérdida de tejido o no, en ciertos casos vemos que éste está en cantidad normal o incluso tiene la potencialidad de desarrollar estructuras dobles, que encuentran una limitación espacial para expresarsc. Un defecto en el desarrollo de la cápsulia nasal, permitiria el descenso de las vesiculas primordiales del cerebro y ocupación de este espacio. Esto produce el cráneo bifido oculto (cubierto por duramadre y periostio) y la alteración de la ubicación de las estructuras faciales. La implantación en $\mathrm{V}$ del pelo en la linea media frontal, se debe a que las zonas perioculares que determinan supresión del crecimiento del pelo, están emplazadas lateralnente. Las muescas laterales en las atas nasales se explican por falla en la fusión de los segmentos laterales y medio de la cresta nasal ${ }^{8-6-7}$.

En algunos casos este sindrome se comporta como una secuencia: hay una interrupción en el desarrollo óseo que Ileva a una protrusión del neurocráneo; el hipertelorismo y las demás mani- 
festaciones son consecuencia del encefalocede anterior ${ }^{9-10}$.

Entre los factores patogénicos que se mencionan están: alteración en el índice initótico y muerte celulas prematura, disminución en la irrigación de la región comprometida por afección de la red vascular embrionaria por algún estimulo teratogénico.

El síndrome de fisura media del rostro, según Sedano y otros autores, ${ }^{12-19}$, tiene ciertos criterios diagnòsticos mínimos que son: hipertelorismo ocular ${ }^{11}$, puente nasal ancho, surco medio nasal o ranuras on alas nasales y ausencia de la punta de la nariz. Por la severidad variuble del sindrome se ha clasificado en 4 tipos: Tabla 1.

Tabla 1

Sindrome de lisura Media del Rostro

Clasificacionn de Sedano 1970

A. HLPT:RTEIORISMO OCIJLAR

PULNTY: NASAL ANCHO

SURCO MEDIO NASAL CON AUSTENCIA DI LA PUNTA DE LA NARIZ

NO HAY VIIRDADFRAS FISURAS MLDIAS

B. HIPERTLLORISMO OCULAR

PULNTE NASAL ANCHO

SURCO MFDIO FACIAL PROFUNDO O VIRDADERA HISURA QUE COMPROMLTT NARIZ O NA. RL $Z$ Y LABIO SUPERIOR

IISURA PALATINA PRLSENTF. IN OCASIONI'S

C. HIPERTELORISMO OCULAR

PUIINTE NASAL ANCHO

MLTESCA EN UNA O AMBAS ALAS NASALLS

D. COMBINACION DE B Y C

Se describen signos fisicas menos comunes que se asocian: braquicefalia, microftalmia, dermoides epibutbares, apéndices de piel preauriculares, senos frontales hipoplásicos y en un $20 \%$ retardo mental en grado leve. La longevidad no estaría comprometida.

En nuestros casos vemos que ef 1 sería el más completo. Sus facies recuerda a un embrión de 45 días. En él hay una duplicación de las estructuras medias, que probablemente determinaron el no desarrollo normal del ojo derecho. 20-21-22 Correspondería a facies $\mathrm{D}$ de Sedano.

Los casos 2 y 3 presentan un defecto en el desarrollo de la cápsula nasal. El 3 no ienía cartílago al nacer y ello seria determinante del resto del cuadro. No es posible a la edad actual ver la presencia de cráneo bifido oculto, pues en seguimientos radiológicos se describe una corrección completa y espontánea. ${ }^{23}$ Ambos corresponderian a facies B de Sedano.

El caso 4, que se acompaña de retardo mental, sociación descrita en este síndrome, presenta una expresión leve con hipertelorismo y una fístula del dorso nasal. Facies A de Sedano.

El caso 5 , seria el más cercano a la normalidad, con una distancia interorbital que casi alcan7a valores normales para su edad y un surco na. sal. En él el retraso psicomotor es atribuible a su prematurez $y$ asfixia neonatal.

Sería importante discernir la forma de herencia de este síndrome, pensando en el consejo genético para elíos y su familia, debido a su integración complera a la sociedad gracias a los trata. mientos que se hat ido desarrollando en cirugia plástica y reparadora.

Su aparición es esporadica y la asociación con otras anomalías es tan variada y ocasional, que parece ser fortuita ${ }^{27-28}$ En la literatura se describen casos que sugieren un tipo de herencia dominante con mutaciones recientes en los primeros propósitos 18.24 . En nuestros pacientes, los padres y hermanos son normales. También se han descrito familias en las que el defecto se transmitía como un rasgo autosómico recesivo; no podemos descartar esta torma de herencia en ruestros casos, a pesar de que no hay consanguinidad en. tre los padres $25 \cdot 26$. No hemos encontrado en lo publicado, casos asociados a alteraciones cromo. sómicas. Todos nuestros pacientes tuvieron cariotipo normal.

Se describen rasgos aislados del sindrome, tales como hipertelorismo o un frenillo labial doble $29-30$, lo que representaría una forma incompleta del cuadro. En nuestros casos, dos familiares lejanos, no examinados por nosotros, tienen labio y paladar fisurado. Sin embargo, el grado de consanguinidad que comparten con los propósitos es tan reducido $\left(4^{\circ}\right.$ o $\left.5^{\circ}\right)$, que su asociación hereditaria es no mayor que la esperada en la población general ${ }^{31-32}$.

Habria una complicada interacción entre susceptibilidad genética para desarroliar la alteración y factores exógenos en el ambiente intrauterino, que juntas y sumadas darían la gama completa del sindrome de fisura media del rostro. La for. ma de herencia poligénica de etiología multifac. torial sería una posibilidad.

\section{RESUMEN}

El síndrome de fisura media del rostro es un cuadro caracterizado por hipertelorismo ocular, ensanchamiento del puente nasal, fisura medio facial que puede comprometer el dorso nasal, el 
labio superior y el paladar, cráneo bífido oculto y otros defectos.

Se presentan cinco casos no relacionados en quienes se ha hecho este diagnóstico y en los que se ha realizado investigación genealógica y estudio cromosómico.

Hemos podido descartar un defecto cromosómico; las genealogías no sugieren un modo de herencia dominante y no excluyen un defecto autosómico recesivo. Es posible que la génesis del sindrome sea multifactorial.

\section{REFERENCLAS}

1 Julio Nazer: Manual de Perinatología. Malformaciones congénitas. Hosp, J.J, Aguirre, Maternidad. Tabla pág. 219.

2 H. Sedano, R. Gortin: Genética craneofacial. Carlos Salinas OPS Cap. 13 Fisuras faciales.

${ }^{3}$ F. Beck, D.B. Moffat, J. B. Lloyd: Human Embriology and Genetics. Chapter 18. The Branchial Region. Mouth, Palate, Nose and Face.

4 Ray F. Stewart: The Pediatric Clinics of North America, August. 1978 Medical Genetics. Craniofacial Malformations: Clinical and Genetic Considerations.

s Hamilton, Boyd, Mossmen: Embriología Humana, 4a. edición, 1973.

' Wiemer, D.: Hardy, S.; Spiro M.: Anatomical Findings in Median Cleft of Upper Lip Plast Reconstr. Surg. 62: 866, 1978.

7 Ponniah, R.: Midline cleft lip with associated abnormalities 3. Laryngol, otol. 97: 177, 1977.

Schwenckendiek, W.: Nasal Abnomalities in facial clefs. J. Maxillofac Surg. Sep. 4: 141, 1976.

${ }^{9}$ Sakodo, K.: Ishikawa, S.: Uozumi, T.; Hirokawa, K.; Okasaki, H., Harada. Y.: Sphenoethmoidal meningoencefalocele associated with agenesis of corpus callosum and median cleft lip and palate. Case report. J. Neurosurg. 51: 397, 1979.

10 Goldahammer, Y.: Smith, $L_{\text {: }}$ Cryptophthalmos syndrome with basal encephaloceles am J. Ophtalmol 80: 146, 1975.

1 I D.W. Smith: Recognizable Patterns of Human Malformations 1970 Tables of Inner Canthal and Outer Orbital Dimensions. Tables of Interpupillary Distances.

12 O. Rogers.: Clinics in Plastic Surgery. An International Quaterly: Cranjofacial Defomities Blair. Vol. 3 No 3: October 1976.

13 Sedano. H.O., Cohen M.M., Jirasek, J., Gorlin, R.J.: Frontonasal Dysplasia. J. Pediat. 76: 906, 1970.
14 Daniel Bergsma.: Birth Defects Compendium. National Foundation-March of Dimes 504 Hypertelorism (Greig Syndrome) 635 Median Cleft Face Syndrome.

15 P.E. Becker.: Genética Humana Tamo II Hiperteloristo de Greig.

16 Rohasco $S . A$., Massa S.L.: Frontonasal Syndrome Brit. 1. Plast. Surg. $31: 344,1968$.

17 Gellis, S.S., Feinglod, M.: Median Cleft Face Syndrome. Am. J. Dis. Child. 127: 85, 1974.

18 Francesconi, G., Fontunato, G.: Median Dystraphia of the Face Plast. Reconstr. Surg. 43: 481, 1969.

19 De Myer, W: The Medjum Cleft Face Syndrome, Differential diagnosis of cronfum bifidum ocuitum, hypertelorism and median cleft nose, lip and palate. Neurology (Minneap) 17: 961, 1967.

20 Feiler, Ofn; God al, V., Nemet, P., Lazar, M.: Retinal Detachment in median Cleft Face Syndrome. Br. J. Ophtalmol 64:-121, 1980.

21 Rintak, A., Unspaa, $Y_{.:}$Anophtalmia and agenesis of columnelk, prolabium and premaxilla without hypotelorism- a New syndrome? Case reports Scand. J. Plast Reconstr. Surg. 12: 69, 1978.

22 Kinsey, J.A., Steeten, BW: Oculat Abnormalities in the median cleft face syndrome Am. J. Ophtalmol 83: 261,1977

23 Kurlander, G.F., De Myer W., Campbell, J.A.: Roentgenology of the Median Cleft Face Syndrome Ratiology 88: 473, 1967.

24 Hootnick, D. and Holmes, L.B.: Familial Polysyn dactyly and Craniofacial Anomalies Clinical Genetics 3: $128,1972$.

25 Glenz, $S$ : Hypertelorism and the Bifid Nose. Southem Medical Journal 59: 631, 1966.

26 Boo Choi, K.: The Bifid Nose with a Report of 3 cases in siblings Plast Reconstructive Surgery 36: 626,1965 .

27 H. Moreno, Fuenmayor: The Spectrum of Frontonasal Dysplasia in an inbred Pedigree. Clinical Genetics 17: 137, 1980 .

28 Arne F. Bakken, Gunnar Asbyholm: Fiontonasal Dysplasig. Possible Hereditary Connection with other congenital defects. Clinical Genetics 10: 214 , 1976.

29 Sharma, L.K.: Median Cleft of the upper Ljp Plast. Reconstr. Surg, 53: 155, 1974.

30 Millard, D.R., Willioms, S.: Median lip clefts of the upper lip Plast Reconstr. Surg, 42: 4, 1968.

31 C.O. Carter: Polygenic Inheritance and Common Diseases. An ABC of Medical Genetics. Chop. 6, p. 50.

32 C.O. Carter: Chap. 19. Multifactorial genetic disease. Medical Genetics. Víctor Mc Kusick-Robert Clajborne 1973 .

${ }^{3}$ Grounchy $J$, Turleau $C$ : Clinical Atlas of Human Chromosomes. Appendix I, Techniques, John Wiley and Sons, N.Y.: $270,1977$. 